\title{
SIGNIFICADO DE LA ENFERMEDAD, EXPERIENCIAS Y EXPECTATIVAS DE PACIENTES CON MIELOFIBROSIS
}

\author{
MEANING OF ILLNESS, EXPERIENCES AND EXPECTATIONS OF PATIENTS WITH \\ MYELOFIBROSIS
}

María Elena Alarcón-Ovalle $e^{1,2}$, José Francisco González-Puentes ${ }^{1}$ y Catalina VelandiaAlarcón

Universidad El Bosque, Bógota, Colombia

2 Organización lberoamericana de la Seguridad Social - OISS

Resumen

Fundamentos: La mielofibrosis es una enfermedad rara que afecta personas adultas especialmente sobre la cual no se encuentran estudios que investiguen el sentir del padecimiento en los pacientes o que aborden lo que ha representado esta enfermedad en la vida El trabajo buscó determinar el significado, las experiencias y las implicaciones que la mielofibrosis tiene en pacientes colombianos.

Métodos: Estudio cualitativo de 10 pacientes con mielofibrosis. Muestreo teórico intencionado. Tecnica: entrevista a profundidad realizada por psicóloga clínica con cuestionario semiestructurado diseñado por los investigadores. Entre diciembre 2013 - enero 2014. Análisis de contenido con apoyo del software Atlas ti. Versión 7, licencia educativa multiusuario.

Resultados: 7 hombres, 3 mujeres con edades entre 19-80 de varias regiones del país, con diagnostico entre 1-17 años. Edad mediana 63.5 años. Cinco con diagnóstico inicial de anemia, leucemia o hepatomegalia. Ninguno conocía previamente la enfermedad. Atribuyen la causa: cocinar con leña, manipulación de materiales en su trabajo, vida agitada sin buena alimentación o razones divinas. El padecimiento de mielofibrosis cambio las concepciones sobre la vida, sus expectativas, hábitos y costumbres. Tienen la convicción de
Abstract

Background: Myelofibrosis is a rare disease on which no studies about the suffering of patients who have it have been conducted. this work seeks to determine the significance, the experiences and the implications that Myelofibrosis has in Colombian patients.

Methods: This is a qualitative study method directed to Myelofibrosis diagnosed patients where theoretical intentional samples were collected. To such end, 10 interviews were conducted between December 2013 and January 2014 by a Clinical Psychologist with semi-structured questionnaires carefully designed by the researchers. The content analysis was developed with Atlas.ti ${ }^{\circledR}$ software support, multi-user educative license, version 7 .

Results: 7 male and 3 female patients between 19-80 years old from various regions of the country were enrolled whose illness had been diagnosed within the 1 and 17 years from the onset of symptoms. Five of the patients had an initial diagnosis of Anemia, Leukemia or Hepatomegaly. None of them knew about their disease previously. They attributed the cause to such symptoms to factors like cooking with firewood, the handling of materials at their work, busy life with poor eating habits or to spiritual reasons. Suffering from the illness has substantially changed the patients' conceptions about life and therefore their expectations,

Correspondencia:

Maria Elena Alarcón Ovalle Universidad El Bosque Avda.

9 No. 131A - 02

Bogotá. Colombia

E-mail: mariahalarcon@gmail.com

El presente trabajo fue financiado por el Laboratorio Novartis quien no participó en ninguna fase del estudio. Los autores declaran no tener conflictos de intereses. 
vivir con apoyo del sistema de salud. La familia, la religión y los médicos se convierten en su apoyo.

Conclusiones No conocían sobre enfermedad. Manifiestan emociones y sentimientos de incredulidad, angustia, tristeza, miedo entre otros y es la familia, la creencia en Dios y la cercanía y comprensión del médico la mejor red de apoyo. Su expectativa frente a la enfermedad es de esperanza.

Palabras clave: Enfermedad rara, padecimiento, investigación cualitativa, mielofibrosis.

\section{INTRODUCCIÓN}

La mielofibrosis es un cáncer hematológico que usualmente se presenta en adultos mayores, con edades entre 50 y 60 años, y síntomas como: fatiga, sudoración nocturna, rasquiña en la piel, dolor óseo, pérdida de peso, fiebre, entre otros. La enfermedad produce una cicatrización de la médula ósea que genera niveles anormales de células sanguíneas y crecimiento significativo de tamaño del bazo. Simultáneamente se generan elevados niveles de citoquinas (sustancia presente en la sangre), que provienen de la alteración de la producción de las células sanguíneas ${ }^{(1)}$.

La mielofibrosis está clasificada como enfermedad rara, poco conocida y subdiagnosticada. Tiene un impacto fuerte en la calidad de vida de quien la padece, a causa de sus signos y síntomas. En los Estados Unidos, entre 2001 y 2004, la enfermedad tuvo una incidencia de 0,2 a 0,5 por 100.000 hab/año y una prevalencia de 2,7 por cada 100.000 individuos ${ }^{(2)}$. Los costos del tratamiento actual de la enfermedad no se han establecido y los desenlaces clínicos son poco evaluables. Por ello, es necesario generar concientización, en torno a la enfermedad, tanto en los pro- habits, and customs. They have the conviction to continue living with the support of the health system and to overcome the disease. Family, religion, and doctors become their support.

Conclusions: They did not know anything about the illness previous to the diagnosis. However, they express emotions and feelings of disbelief, anger, sadness, and fear among others and it is the family, the belief in God and the closeness and understanding of their doctors which constitutes their main support network. Hope is the only expectation he has towards the disease.

Keysword: Rare disease, stress psychological, qualitative research, myelofibrosis.

fesionales de la salud como en los comités que toman decisiones en las empresas prestadoras de salud ${ }^{(1)}$.

El plan decenal para el control de cáncer en Colombia 2012-2021(3) establece en la línea estratégica número 4 el "mejoramiento de la calidad de vida de pacientes y sobrevivientes con cáncer". En el que se plantea la atención integral al paciente oncológico, inclusive a su familia. Igualmente, se propone como una acción a nivel político y normativo, la realización de mediciones relacionadas con la calidad de vida de las personas que tienen la enfermedad y las que han sobrevivido. En este contexto, llevar a cabo estudios que favorezcan esta intención justifica la realización del presente estudio, que tiene como objetivo determinar el significado y las expectativas que tienen sobre la enfermedad pacientes diagnosticados con mielofibrosis en relación con sus experiencias.

\section{SUJETOS Y MÉTODOS}

El tipo de estudio fue cualitativo exploratorio, con dos niveles de análisis: descriptivo e interpretativo. La duración del estudio fue de siete meses (septiembre del 
2013 a marzo 2014). Se pueden distinguir dos momentos en la fase de trabajo de campo: uno relacionado con el acercamiento a los médicos tratantes para identificar los pacientes objeto de estudio y el otro el establecimiento de contacto con los pacientes para realizar la entrevista. En el primer momento, se obtuvo información de 117 profesionales especialistas en oncología, hematología y hemato-oncología, quienes fueron contactados telefónicamente o por correo electrónico. 29 de estos reportaron atender en ese momento pacientes diagnosticados con mielofibrosis. Se procedió a enviarles copias del proyecto, del acta del comité de ética y del consentimiento informado para que conocieran detalles de la investigación. Ellos consultaron a los pacientes la posibilidad de asistir y dar una entrevista. Después que los pacientes aceptaron la invitación de los médicos, se inició el acercamiento con cada uno de ellos. No todos los médicos pudieron localizar a los pacientes que tenían en sus historias.

El segundo momento implicó llamar por teléfono a los pacientes para explicarles en qué consistía la entrevista y para responder las dudas que tuviesen. Se contactaron 11 pacientes de los cuales 10 aceptaron participar y en la tabla 1 aparecen las características sociodemográficas.

La entrevista a profundidad se efectuó por medio de un cuestionario semiestructurado el cual se presenta en el anexo. La profesional encargada para tal fin fue una de las investigadoras psicóloga clínica, quien, previo consentimiento informado, grabó en audio cada una de las entrevistas. Los datos obtenidos se transcribieron textualmente en el procesador de textos Word, pregunta por pregunta, con todas las ex-

\section{Tabla 1. Características sociodemográficas de los pacientes del estudio}

\begin{tabular}{|l|l|}
\hline Edad en años & Entre 19 - 80: hombres 40 - 80 y mujeres 19 - 65. Edad mediana $=63,5$ \\
\hline Sexo & 7 hombres y 3 mujeres \\
\hline Lugar de residencia & $\begin{array}{l}8 \text { viven en capitales de departamento: Cali (2) Bogotá (4), Ibagué (1), } \\
\text { Riohacha (1) y dos en municipios, Fusagasugá, (Cundinamarca) (1) y La } \\
\text { Capilla (Boyacá) (1). }\end{array}$ \\
\hline Estructura familiar & $\begin{array}{l}\text { En las mujeres: una es soltera vive con su padre, madrastra y hermano } \\
\text { medio, una separada y vive con una de sus hijas casada y la otra tuvo dos } \\
\text { relaciones afectivas pero hoy vive con uno de sus hijos y una hermana. } \\
\text { En los hombres: 6 residen con su esposa (en dos casos es la segunda } \\
\text { esposa) y algunos con uno de sus hijos. Uno es viudo y vive con una her- } \\
\text { mana. Solamente uno de ellos no tiene relación cercana con sus hijos. }\end{array}$ \\
\hline $\begin{array}{l}\text { Actividad laboral en } \\
\text { el tiempo }\end{array}$ & $\begin{array}{l}\text { Hombres: comerciante, ejecutivo, médico-profesor de medicina, cafete- } \\
\text { ro, constructor, vendedor de seguros y mensajero-almacenista. } \\
\text { Mujeres: una fue diseñadora de modas, otra ama de casa y la más joven } \\
\text { estudiante. }\end{array}$ \\
\hline Actividad actual & $\begin{array}{l}\text { Siete retirados, algunos por la enfermedad otros están pensionados, tres } \\
\text { ocupados un vendedor de seguros, un constructor que según su condi- } \\
\text { ción de salud puede asistir y la paciente más joven, trabaja en ventas en } \\
\text { épocas de vacaciones. }\end{array}$ \\
\hline $\begin{array}{l}\text { Condiciones econó- } \\
\text { micas (no se preguntó) }\end{array}$ & $\begin{array}{l}\text { Tres de ellos tiene estrato socioeconómico alto, seis son de estrato so- } \\
\text { cioeconómico medio y uno de ellos es de estrato bajo. }\end{array}$ \\
\hline
\end{tabular}


presiones, palabras, sonidos, (llantos, risas, etc.). Los textos fueron leídos y analizados por cada uno de los investigadores con el fin de identificar categorías y subcategorías de acuerdo con los objetivos de estudio y la literatura que se encontró para padecimientos crónicos en pacientes ${ }^{(15,16,20)}$ : reacción al diagnóstico, redes de apoyo, condición de la enfermedad, significado de la enfermedad. A la vez, los textos fueron procesados en el programa de análisis cualitativo Atlas.ti ${ }^{\circledR}$ y sometidos a un proceso de codificación que permitió visualizar, de manera gráfica y por subcategorías, los resultados. De este modo, se analizó el conjunto de significados, experiencias y expectativas de los pacientes en relación con su enfermedad como un sistema completo y articulado. Con los análisis de los investigadores y lo encontrado en Atlas.ti, se determinaron finalmente las categorías y subcategorías de análisis

\section{RESULTADOS}

A continuación se presentan los resultados obtenidos, de acuerdo con los objetivos y el planteamiento metodológico expuesto.

Se encontró que el tiempo de diagnóstico de la enfermedad fue variable. Se identificaron diagnósticos entre 1 y 17 años, con una mediana de años-diagnóstico de 3,5 años. Sin embargo, el proceso para llegar al diagnóstico no fue fácil; algunos pacientes han tenido que vivir años de exámenes y dificultades, como el traslado a la capital, hospitalizaciones, intervenciones quirúrgicas o tratamientos. Cinco de los diez pacientes entrevistados tuvieron un diagnóstico inicial de otras enfermedades como anemia, leucemia o hepatomegalia. Una de las pacientes, por ejemplo, recibió tratamiento para leucemia durante siete años. Ella viajó a Bogotá después de asistir a una visita "donde un señor que cura a través de plantas naturales", y quien después de revisarla le halló "una bola, una masa" y le sugirió que la llevaran a una clínica. Cuatro pacientes refirieron tener otras enfermedades, como diabetes, hipertensión y problemas cardiacos.

En relación con el significado de la enfermedad, se indagó el conocimiento previo que tenían los participantes sobre la mielofibrosis. Ninguno de ellos tenía conocimiento previo, incluso el participante profesional en el área de la salud se expresó al respecto: "Eh... no, yo que iba a conocer de eso, nada"; "No, no tenía ni idea, ni idea que eso existía".

Se encontró que los pacientes consideraban como causa de la enfermedad la exposición a ciertos factores de riesgos como: cocinar con leña, (su médico lo había informado), el uso de pegantes como material de trabajo, la vida agitada y otros la asocian con razones divinas. "Los pegantes, yo siento que a mí me afectaron..."; "Yo le echo la culpa más... una vida muy agitada..."; ...A Chucho (Dios)... que yo que he hecho, pues santo no soy pero malo tampoco..."

El diagnóstico de cáncer tiene efectos sobre el individuo. Para los participantes del estudio conocer el diagnóstico provoca una reacción de tipo emocional, que pasa por la angustia, la tristeza, el miedo, la incredulidad, la evasión hasta la preocupación. Además, la asociación cultural entre el cáncer, la muerte y el dolor de la familia, por su posible fallecimiento, se manifiesta. En todos los casos los pacientes acudieron a una creencia religiosa o espiritual para sobrellevar la situación. "Me sentí muy mal después me sentí peor..."; "...eso se lo entregaba a Dios..." "...hay veces que me pongo como a pensar en cosas que no debo pensar, pero a la hora de la verdad, eso Dios, que es el dueño de mi vida,..." (Llanto).

Dos pacientes refirieron estar tranquilos con el diagnóstico, pues sus médicos les ofrecieron una opción de tratamiento. 
"...No me angustié, no me angustié porque me comentaron que no era, que no era una leucemia ...que era un problema tratable..."; "...cuando me dijo eso yo pensé, bueno pues son cuatro o cinco años, ...de aquí a eso algo habrán descubierto..., pero que me hubiera trasnochado ini cinco!". Después de conocer el diagnóstico, dos les suscito cierta curiosidad: comenzaron a buscar información sobre el tema. "... En ese momento no nos impactó mucho, ya después cuando empecé a leer e investigar...".

La reacción de la familia, en el momento del diagnóstico, tuvo similares respuestas emocionales: tristeza, miedo, entre otros. Los pacientes repiten la palabra "duro" y afirman: "...Pues a mi mamá y a mi esposa pues les dio durito,...no... pues fue muy tremendo tremendo tremendo, los bebes muy pequeños y todo"; "entonces pa' nosotros fue duro, tenso, duro duro..." "...pues fue dura, sí porque, no sé si era como un cáncer, o algo yo no sé..."

Frente a los cambios en las relaciones con pares, se encontró que algunos no hablan de su enfermedad, para no generar lástima de ellos y sentir vergüenza. Otros manifiestan que ya no pueden salir de sus casas. Además, dicen, a quienes antes consideraban amigos, ahora los perciben como simplemente compañeros: "... Siento pena, no me gusta, porque entonces una persona que se siente enferma, que tiene esta enfermedad...; "...los amigos eran cuando podía tomar, podía salir..." Solamente uno de ellos siente que sus amistades son también apoyo en el manejo de su enfermedad.

La enfermedad generó cambios en la persona, la familia, el trabajo y la sociedad. Se evidenciaron diferentes posturas frente a la vida social. Algunos participantes se alejaron de la vida social que llevaba "A raíz del diagnóstico a mí se me acabó la farrita (parranda) se acabó todo...". En general, tuvieron que cambiar hábitos: "Me volví muy sedentaria, yo no era así...". Incluso un paciente hablaba de no poder ir a trabajar por la enfermedad: "Pues, no podía trabajar,... ahora me afecta de todo." Otro participante se refiere al trabajo como un factor de protección: "Mis rutinas no cambiaron...como tenía más responsabilidades, tenía que estar más inmerso en el trabajo..." Por último, uno de ellos no identificó cambios en su vida: "Yo seguí la vida como si no la tuviera...".

Algunos admiten modificaciones en sus hábitos alimenticios. Después del diagnóstico, estos han dejado de comer lo que estaban acostumbrados y han incrementado comida más "sana" como verduras y frutas, según explican. "No, pues de pronto ahora me cuido más con la alimentación, mucho más... Como pues mucha verdura..."; "...cambie más dramáticamente hábitos,... las harinas las reduzco, si voy a comer algo de dulce yo sé... en poca cantidad". Otros han continuado con su alimentación sin ningún cambio. Yo sigo comiendo igual..."; "...la alimentación tampoco, tampoco cambió".

La expectativa frente a la enfermedad, para varios de ellos es de esperanza. Con la aparición de un medicamento, más de la mitad de los pacientes lo reconocen, como un gran avance que les permitirá vivir mejor, mientras lo estén consumiendo. Cabe resaltar el caso de dos pacientes que contemplan la curación de su enfermedad: "Yo abrigo la esperanza de...seguir viviendo como hasta el momento...";"... mis expectativas no, pues que tengo que tomarme juiciosa la pasta...para estar bien...". En dos voces se escucha la desesperanza; no hay optimismo, hay incertidumbre: "No, no hay expectativas,..."; "...ya vino el día de hoy y espero el de mañana". Dos participantes manifiestan la esperanza de curarse: "...Pero yo creo que es curable,..."; "...pues de pronto lograr un trasplante..., eliminar totalmente la enfermedad...". Para la mayoría, (excepto 1) 
Dios y la fe se convierten en aliados para alcanzar lo que desean. Dicen: "Dios, que es el dueño de mi vida..."

Todos manifiestan que quieren realizar actividades, que les gusta estar "activos", no necesariamente en el trabajo; es decir, buscan constantemente en qué ocuparse: "Quieto no me estoy... cuando estoy así me pongo a leer el periódico"; "...Sí, yo estoy activa todo el tiempo, me gusta... estar pilas...".

Con respecto a su condición de salud, relacionada con la mielofibrosis, los pacientes de este grupo manifiestan que no los afecta de manera negativa: "Porque a mí no me duele nada, pues yo estoy bien...". Incluso, los que tienen otras enfermedades expresan: "Soy diabético y me aplico insulina. Tomo droga para la hipertensión. Pero en general bien: duermo como un bebé, como bien y no tengo fatiga...". "Me siento mejor, sobre todo ahora que me cambiaron el medicamento...". Dos pacientes -la persona más joven y el paciente con mayores dificultades de acceso- manifiestan incomodidades en su condición actual de salud malestar estomacal y dolor de cabeza.

En la mayor parte de los casos, las redes de apoyo se establecen en la familia (la esposa o los padres). Igualmente, el factor religioso es determinante frente a la enfermedad, es una forma de sentirse respaldado y de mantener la lucha por la vida: "...Sí, soy muy religioso;...creo que debería tener más fe, de la que tengo"; "...si claro, porque sin Él (Dios) pues ya estaría en otro lado".

La buena relación con el médico tratante, e incluso la cercanía con el profesional, emerge como una categoría en las redes de apoyo a los pacientes: les da seguridad, tranquilidad y confianza. La mayoría -nueve- de ellos refieren que los médicos procuran mantenerlos actualizados sobre la enfermedad y los apoyan en el proceso de adquisición de medicamen- tos y la programación de citas médicas. Los describen como personas cercanas, con gran calidez humana, que brindan un trato adecuado y procuran mejorar la calidad de vida. "Con la doctora, que es una adoración de mujer, es una profesional excelente, adoradísima, que a todos nos ayuda"; "...la doctora de la clínica es como, como una mamá...tengo buena relación, no sé si ellos son diferentes con todos los pacientes, pero uno siente cierta calidez..."; "...la doctora... una bendición de Dios...". Uno de los pacientes expresa que no siempre cuenta con la suerte de tener una cita con el médico que le gusta: “... La esperanza mía era que me atendiera el médico... pero estaba la agenda tan copada...". Este mismo paciente, manifestó descontento con los médicos que lo habían tratado, pues consideraba que no le habían formulado el "remedio" apropiado. Algunos de ellos buscan soporte en otras actividades como la práctica del yoga, el ejercicio diario o practican el canto: "... Ahora estoy tomando yoga y hace poco me vinculé a un coro y tomo a veces cursos..." "... a mí me gusta hacer ejercicios de yoga por la mañana..."

\section{DISCUSIÓN}

Como enfermedad "rara", la mielofibrosis ha sido poco estudiada en diferentes niveles de análisis, (4). La mayoría de los estudios se centran en el comportamiento y manejo de la enfermedad con enfoque epidemiológico, y no se encuentran investigaciones que recojan la perspectiva de las personas que la padecen.

Los pacientes del estudio tenían una edad mediana de 63,5 años lo cual es similar a los resultados en otros estu$\operatorname{dios}^{(4-7)}$ quienes reportaron en sus trabajos pacientes con edad entre 59 y 63 años. La mitad de los pacientes fueron diagnosticados con anemia inicialmente al igual que otros estudios ${ }^{(4,11)}$. 
Con respecto a las demás categorías analizadas, la contrastación teórica que se realiza a continuación está basada en estudios sobre cáncer o enfermedades crónicas.

En relación con la reacción frente a la enfermedad, se evidenció ambigüedad frente a cómo se sentían por la noticia ${ }^{(13)}$ De acuerdo con Hueso et al, (15) el diagnóstico generalmente se recibe con incredulidad, sufrimiento y angustia, sensaciones que también manifiestan los participantes de la presente investigación (mencionan el miedo, incertidumbre y angustia). De igual forma otros estudios ${ }^{(16,17)}$ identificaron miedo y expectativa frente al tratamiento e incertidumbre ante lo que sucederá.

Así mismo, se encontró que los familiares comparten la presencia de los mismos sentimientos. Habitualmente el familiar más cercano es quien asume la mayor parte de responsabilidad del cuidado ${ }^{(15)} \mathrm{De}$ acuerdo con Solano y Siles ${ }^{(18)}$, generalmente la persona que padece la enfermedad considera que es una carga para su familia, de modo que se crea en el sujeto una percepción de dependencia que contribuye a incrementar su malestar y a alterar las relaciones familiares. Si bien en el presente estudio se identificó que había un incremento en el malestar, no se evidenció alteración en las relaciones familiares, tal como lo formula Garcia ${ }^{(16)}$ quien explica que existe una fragmentación familiar y dificultad en las relaciones posterior al inicio de la enfermedad, por el contrario, se encontró que la familia es el principal apoyo en los diferentes momentos de la enfermedad, tanto en lo físico como lo emocional. Lo mismo ha sido encontrado en estudios ${ }^{(14,19)}$ o incluso mayor cohesión como en otro estudio ${ }^{(15)}$.

Frente a los cambios en su estilo de vida, después del diagnóstico de mielofibrosis, se identificaron cambios en todos los participantes tanto en lo laboral como en lo social. En unos más significativos que en otros. Esta información se corrobora con lo encontrado en estudios ${ }^{(15,16,20)}$ quienes afirman que los cambios físicos y emocionales de las enfermedades producen cambios no solo en la actividad diaria sino en la vida social y la actividad laboral. Específicamente en el contexto laboral, se identificaron cambios conflictivos en los participantes, principalmente la imposibilidad de tener trabajos estables, debido a las condiciones de salud cambiantes. Esta situación impide que la persona tenga un desempeño adecuado en su actividad laboral(22).

Algunos participantes reconocieron que no era necesario comunicar su situación a pares, pues probablemente sentirían lástima por ellos, lo cual en el estudio de Fons et $\mathrm{al}^{(19)}$ se denominó como estigmatización del "enfermo".

En cuanto a la atención en los servicios de salud, un participante reportó inconformidad lo cual coincide con ciertos estu$\operatorname{dios}^{(15,21)}$, mientras que frente a la relación con los médicos los pacientes tienen una percepción de confianza y respeto mutuo igual a lo manifestado en otra investigación $^{(15)}$.

En conclusión, a pesar de los momentos de desesperanza e incertidumbre que se presentan en los pacientes, en términos generales, las expectativas frente a la vida es la de subsistir más años y, en el mejor de los casos, curarse de la enfermedad. A pesar que los pacientes no expresan cambios significativos en su vida por causa de la enfermedad, al profundizar en el análisis de los relatos brindados, se encuentran cambios en varios aspectos de la vida, tales como el trabajo, las relaciones familiares y en ciertas conductas asociadas con el cuidado de la salud, la alimentación, la rutina del sueño y la actividad física entre otros.

La muerte para algunos es un hecho inevitable y han aprendido a vivir con la incertidumbre de no saber cuándo suce- 
derá. La vivencia de la enfermedad afecta no sólo a la persona sino también a la red familiar. En algunos casos, el padecimiento lo enfrentan los seres más cercanos (la esposa o los padres). Estos sufren en silencio y buscan que el paciente no se entere de lo que piensa o siente el cuidador. La familia es el principal apoyo para los pacientes al igual que la religión y el sistema de salud especialmente su relación con el médico tratante, hacen más Ilevadera la enfermedad.

La comprensión del sentir de los pacientes con patologías como la mileofibrosis nos indica la necesidad de discutir sentimientos, compartir su miedo, tristezas e incertidumbre, aliviando tensiones generadas por la situación que genera angustia y temor. El cuidado de estas personas implica el desarrollo de la sensibilidad para observar al paciente como débil, como alguien que requiere no solo cuidados para el cuerpo físico, sino también en otros aspectos de su existencia, por esto se hace necesario que se trabaje en una atención integral que involucre no solo médicos sino psicólogos y psiquiatras entre otros.

\section{AGRADECIMIENTOS:}

A los pacientes por haber dedicado tiempo y haber abierto su sentir para la realización de las entrevistas.

A Novartis, por su aporte económico para la realización del estudio y a Carmen Yadira García, quien con gran profesionalismo nos orientó en cada uno de los aspectos administrativos con la empresa.

A la Universidad El Bosque - Vicerrectoría de Investigaciones - por confiarnos esta tarea y respaldar nuestro estudio.

\section{REFERENCIAS BIBLIOGRÁFICAS}

1. Leukemia \& Limphoma Society Fighting. Información sobre la Mielofibrosis [Internet]. 2012 jul [citado 2013.6]; 14S:1-9.
Disponible en: http://www.lls.org/content/ nationalcontent/resourcecenter/freeeducationmaterials/spanish/pdf/sp_idiopathicmyelofibrosis.pdf

2. Rollison D, Howlader N, Smith M, Strom S, Merritt W, Ries, et al., A. Epidemiology of myelodysplastic syndromes and chronic myeloproliferative disorders in the United States, 2001-2004, using data from the NAACCR and SEER programs. Blood 2008;112:45-52. Doi: 10.1016/j. amjmed.2012.04.014

3. Colombia. Ministerio de Salud y Protección Social e Instituto Nacional de Cancerología, ESE. Plan Decenal para el Control del Cáncer en Colombia, 2012-2021 [Internet]. 2012 [citado 2013 en. 5]. Disponible en:

http://www.minsalud.gov.co/Documentos $\% 20 y \% 20$ Publicaciones/Plan $\% 20$ Decenal\%20para\%20el\%20Control\%20 del\%20C\%C3\%A1ncer.pdf

4. Ávila O, González Y, Espinosa E, Rodríguez L, Izquierdo L, Espinosa E. Aspectos diagnósticos, evolutivos y terapéuticos de la mielofibrosis primaria. Rev Cubana Hematol Inmunol Hemoter [Internet]. 2011 dic. [citado 2014 en. 6];27(4):40717. Disponible en: http://scielo.sld.cu/ scielo.php?script=sci_arttext\&pid=S086402892011000400005\&lng=es

5. Mesa, R, Chin-Yang L, Ketterling Rhett $P$, Schroeder Georgene S, Knudson A, Tefferi A. Leukemic transformation in myelofibrosis with myeloid metaplasia: a single-institution experience with 91 cases. Blood 2005;105:973-7. Doi:10.1182/ blood-2004-07-2864

6. Tefferi A. The forgotten myeloproliferative disorder: Myeloid metaplasia. Oncologist Oncologist. 2003;8:225-31. Doi: 10.1634/ theoncologist.8-3-225

7. Anderson, J, Tefferi A, Craig, F, Holmberg, L, Chauncey T, Appelbaum F. Myeloablation and autologous peripheral blood stem cell rescue results in hematologic and clinical responses in patients with mye- 
loid metaplasia with myelofibrosis. Blood 2001; 1;98:586-93. Doi:10.1182/blood. V98.3.586

8. Novales, X, Chávez A. Mielofibrosis en niños. Rev Mex Pediatr. [Internet]. 2002 [revisión 2002 nov-dic; citado 2014 en. 3]; 69 (6):252-56. Disponible en:

http://www.medigraphic.com/pdfs/pediat/ sp-2002/sp026h.pdf

9. Linardi, C, Dalessandro, T, Pracchia L, Buccheri V. Evaluation of survival risk scores in primary myelofibrosis: A Brazilian experience from a single institution. Leuk Lymphoma. 2008;49:1641-3. Doi: 10.1080/10428190802141194.

10. Colombia. Ministerio de Salud y Protección Social. Dirección de Epidemiología y Demografía. Morbilidad atendida en los servicios de Salud y Mortalidad. (2014) [Internet]. [citado 2014 en. 3]. Disponible en: http://www.minsalud.gov.co/sites/rid/ Paginas/results_advanced.aspx?u=http $\% 3$ A $\% 2 F \% 2 F w w w 2$ Eminsalud $\% 2$ Egov $\% 2 \mathrm{E}$ co $\% 2$ Fsites $\% 2$ Frid\&k=mielofibrosis

11. Tefferi A. How I treat myelofibrosis. Blood 2011:31;117:3494-504. Doi: 10.1182/ blood-2010-11-31561

12. Cervantes F, Dupriez B, Pererira A, Passamonti F, Reilly J, Morra E, Vannucchi A. New prognostic scoring system for primary myelofibrosis based on a study of the International Working. Blood 2009;26;113:2895-901. Doi: 10.1182/ blood-2008-07-170449

13. Maruyama S, Zaga, M. O processo de adoecer do portador de colostomia por câncer; Rev. Latino-Am. Enfermagem [Internet]. 2005 Mar-abr. [Citado en 2014. 6];(2):216-22. Disponible en: http://www. scielo.br/pdf/rlae/v13n2/v13n2a13.pdf

14. Hwang, E, Kim, Y, Jun S. Lived experience of Korean women suffering from rheumatoid arthritis: A phenomenological approach. Int J Nurs Stud 2004;41:239-46. Doi:10.1016/S0020-7489(03)00132-9

15. Hueso C, Siles J, Amezcua M, Bonill C, Pastor S, Celdrán M. Comprendien- do el padecimiento humano ante la enfermedad: manifestaciones, contexto $y$ estrategias. Rev Latino-Am Enfermagem 2012;20:619-28. Doi:10.1590/ S0104-11692012000300026.

16. García A. Las trayectorias del padecimiento: hombres adultos amputados y no-amputados a causa de la diabetes mellitus. Invest Educ Enferm [Internet]. 2004 sep. [citado 2014 en. 5];22:28-39. Disponible en: http://www.redalyc.org/articulo. oa?id=105216892006.

17. Paiva L, Rossi L, Costa M, Dantas R. Experiência do paciente politraumatizado e suas consequências. Rev. Latino-Am. Enfermagem. [Internet]. 2010 nov-dic. [citado 2014 en. 5];18(6):1221-28. Disponible en:

http://www.revistas.usp.br/rlae/article/ viewFile/4278/5426

18. Solano M, Siles J. The experience of the coronary patient in critical care. Index Enferm. 2005; 14:29-33. Doi:10.4321/ S1132-12962005000300006.

19. Fons A, Pujol, A, Sánchez A, Feijoo M, Bernat E, Moreno C. Creencias, actitudes y conocimientos de las personas con diabetes tipo 2 respecto a su enfermedad. Enferm Clín 2004;14: 307-12

20. Mercado-Martínez F, Robles L, Ramos I, Leal N, Alcántara E. La perspectiva de los sujetos enfermos. Reflexiones sobre pasado, presente y futuro de la experiencia del padecimiento crónico. Cad Saúde Pública 1999; 15(1). Doi:10.1590/S0102311X1999000100018.

21. Chibaci R, Barbosa M, Yasumori Y. A mulher japonesa vivenciando o câncer cérvico-uterino: um estudo de caso com abordagem da fenomenologia social. Rev Esc Enferm USP 39:189-94.Doi:10.1590/ S0080-62342005000200009.

22. Siqueira K, Alves M, Boemer M. El vivir a situación de ser con cáncer: algunas elucidaciones. Rev Latino-am Enfermagem 2007;15:(4). Doi: 10.1590/S010411692007000400013 


\section{ANEXO \\ INSTRUMENTO DE RECOLECCIÓN DE LA INFORMACIÓN \\ SIGNIFICADO DE LA ENFERMEDAD, EXPERIENCIAS Y EXPECTATIVAS \\ DE PACIENTES CON MIELOFIBROSIS}

1. Nombre

2. Fecha de Nacimiento

3. Edad

4. Ocupación

5. Lugar de Residencia

6. ¿Cómo se compone su núcleo familiar?

7. ¿Con quien vive actualmente?

8. ¿Conocía la Mielofibrosis antes de ser diagnosticado?

9. ¿Hace cuanto fue diagnosticado con Mielofibrosis?

10. ¿Cómo se sintió en el momento de recibir el diagnóstico? ¿Qué fue lo primero que pensó?

11. ¿Esa reacción inicial ha cambiado hasta el día de hoy? ¿Cómo?

12. ¿Desde el momento del diagnóstico, su rutina ha cambiado? ¿Cómo? ¿Podría describir un día normal en su vida?

13. ¿Ha tenido que modificar comportamientos para realizar un adecuado cuidado de su salud? ¿Cómo cuáles?

14. ¿Cuál es la expectativa que usted tiene frente al pronóstico de su enfermedad?

15. ¿Su vida ha cambiado desde el momento del diagnóstico de la enfermedad? ¿En qué?

16. ¿Cuál fue la reacción de su familia frente a su diagnóstico? ¿Y la de sus amigos y compañeros de trabajo?

17. ¿Cuál ha sido el papel de ellos durante su enfermedad?

18. ¿Cuenta con otras fuentes de apoyo? (Grupos religiosos, servicios sociales)

19. ¿Con qué servicio de salud cuenta?

20. ¿Cree usted que el tipo de servicio de salud se relaciona con un adecuado manejo de la enfermedad?

21. Describa su relación con los profesionales de la salud con los que interactúa ¿Esto facilita o dificulta el manejo médico de la enfermedad? 